\title{
Outcomes of prior cervical cytology and HR- HPV testing in women subsequently diagnosed with CIN1, CIN2/3, and invasive cervical cancer: a 4-year routine clinical experience after implementation of systematic training and quality control programs
}

Dongman Zhao ${ }^{1+}$, Liran Zhang ${ }^{1 \dagger}$, Fengxiang Xie ${ }^{1}$, Dezhi Peng ${ }^{2}$, Jie Wei ${ }^{2}$, Lingbo Jiang ${ }^{2}$, Shoudu Zhang ${ }^{2}$ and Debo Qi ${ }^{2 *}$

\begin{abstract}
Background: In 2013, Jinan KingMed Diagnostics (JKD) first established a systematic cervical cytology training and quality control (QC) program in Shandong Province, China. We compared the efficacy of high-risk human papillomavirus (HR-HPV) detection, cytology, and their combination in routine clinical practice after the implementation of the training and QC program to identify the optimal first-line screening method in this region.

Methods: The data of patients histologically diagnosed with cervical intraepithelial neoplasia (CIN) 1, CIN2/3, and invasive cervical cancer (ICC) between January 2014 and December 2017 were retrieved from the JKD database. Cytology and/or HR-HPV testing results within 3 months preceding the CIN1 diagnoses and 6 months preceding the CIN2/3 and ICC diagnoses were analyzed.
\end{abstract}

Results: Prior screening data were available for 1829 CIN1 patients, 2309 CIN2/3 patients, and 680 ICC patients. Cytology alone and HR-HPV testing alone had similar rates of positive results for CIN2/3 (97.2\% [854/879] vs. 95.4\% [864/906], $P=0.105$ ) and ICC detection (89.1\% [205/230] vs. 92.7\% [204/220], $P=0.185)$. Compared with either method alone, co-testing slightly increased the screening sensitivity for CIN2/3 (99.8\% [523/524], all $P<0.001)$ and ICC (99.6\% [229/230], all $P<0.001)$ detection. In the CIN1 group, cervical cytology alone (92.9\% [520/560]) was more sensitive than HR-HPV testing alone (79.9\% [570/713], $P<0.001)$, and co-testing (95.3\% [530/556]) did not significantly improve the screening sensitivity $(P=0.105)$.

(Continued on next page)

\footnotetext{
*Correspondence: bobo8369@163.com

${ }^{\dagger}$ Dongman Zhao and Liran Zhang contributed equally to this work.

${ }^{2}$ Department of Laboratory Medicine, Jinan KingMed Diagnostics, Jinan 250101, Shandong Province, China

Full list of author information is available at the end of the article
}

C C The Author(s). 2020 Open Access This article is licensed under a Creative Commons Attribution 4.0 International License, which permits use, sharing, adaptation, distribution and reproduction in any medium or format, as long as you give appropriate credit to the original author(s) and the source, provide a link to the Creative Commons licence, and indicate if changes were made. The images or other third party material in this article are included in the article's Creative Commons licence, unless indicated otherwise in a credit line to the material. If material is not included in the article's Creative Commons licence and your intended use is not permitted by statutory regulation or exceeds the permitted use, you will need to obtain permission directly from the copyright holder. To view a copy of this licence, visit http://creativecommons.org/licenses/by/4.0/ The Creative Commons Public Domain Dedication waiver (http://creativecommons.org/publicdomain/zero/1.0/) applies to the data made available in this article, unless otherwise stated in a credit line to the data. 
(Continued from previous page)

Conclusions: After the implementation of a systematic training and QC program, both cytology and HR-HPV testing may be adopted for primary cervical cancer screening in Shandong Province.

Keywords: Cervical intraepithelial neoplasia, Cervical cancer, Screening, Cervical cytology, High-risk human papillomavirus (HR-HPV), Co-testing, Quality control, Training

\section{Background}

Cervical cancer is the third most common malignancy in women, and approximately $85 \%$ of all cases of cervical cancer occur in low-resource countries, where there is a lack of trained personnel for cervical cancer screening [1]. Cervical cancer mainly develops from precancerous lesions, namely, cervical intraepithelial neoplasia (CIN) $[2,3]$. The transformation from CIN to invasive cancer generally takes about 5 to 10 years $[2,3]$. If these precancerous lesions are obliterated, the occurrence of most cases of invasive cervical cancer (ICC) can be effectively prevented. Over the past several decades, conventional Papanicolaou smear (CPS) has been used as an efficient, cost-effective screening method for the prevention and early diagnosis of cervical cancer. In the United States and most other developed European countries, systematic training programs for cytotechnologists and/or cytopathologists as well as detailed regulations governing cytopathological quality control (QC) processes are well-established to ensure the efficacy of screening [4, 5]. After the implementation of these training and QC provisions, the incidence and mortality of cervical cancer has drastically reduced in the last 60 years [68]. At the beginning of this century, the cytological efficiency of cervical cancer screening was further improved with the widespread application of liquidbased cytology (LBC) [9, 10].

Persistent high-risk human papillomavirus (HR-HPV) infection, especially with the HPV-16/18 genotypes, is the leading cause of cervical cancer and its precancerous lesions $[2,11]$. Initially, HR-HPV detection was used for the further triage of abnormal cervical cytological results that could not be clearly interpreted [12]. However, clinical studies $[13,14]$ have shown that adding HR-HPV testing to cytology can increase the detection rate of cervical cancer and its precursor lesions in the screened populations. Based on these findings, HR-HPV and cytology co-testing was recommended as the primary screening modality for women aged 30 to 65 years [15]. In recent years, however, several large, randomized controlled clinical studies have revealed that HR-HPV testing alone can detect more cases of cervical cancer and its precancerous lesions than cytological screening [1619]. The ATHENA study has further confirmed that the detection rate of CIN3 and above lesions by HR-HPV testing with separate HPV16 and HPV18 detection was comparable to that of a single cytological screening test [20]. Based on the above results, HR-HPV testing was approved as a first-line method of cervical cancer screening in several developed countries [21, 22].

Cervical cancer is highly prevalent in China. In 2012, Chinese women accounted for $12 \%$ of all new cases and $11 \%$ of all deaths due to cervical cancer in the world [23]. Both the incidence and mortality rates of cervical cancer in China have been showing year-on-year increases since a decade $[23,24]$. Furthermore, due to the lack of a standardized cancer registration system, the cervical cancer incidence and mortality rates in China, especially in suburban and rural areas, might have been underestimated. Although some large-scale cervical cancer screening programs have been carried out in China, there is a lack of qualified cytopathologists, and cervical cancer screening is often not viable in rural or lowresource areas $[25,26]$. Currently, China has not yet established a well-organized training system and unified cervical cytology QC standards for cervical cancer screening $[25,26]$. These factors might have resulted in low screening efficiency of cervical cytology. In recent years, HR-HPV detection alone as well as HR-HPV and cytology co-testing were applied for routine cervical cancer screening in the as yet largely unscreened Chinese population. Presently, three modalities are available for cervical cancer screening: cytology alone, HR-HPV testing alone, and HR-HPV and cytology co-testing. However, no consensus has been reached about which of these is the optimal first-line screening method for cervical cancer prevention.

KingMed Diagnostics (KD) is the largest independent operator of pathology laboratories in China, and has established China's first ever training school for cytopathologists and formulated cytological QC measures at its headquarters in Guangzhou, in accordance with the College of American Pathologists (CAP) requirements [10]. Jinan KingMed Diagnostics (JKD), a local pathology laboratory of KD in Shandong Province, has conducted a similar cytopathologist training and QC program since 2013. After the implementation of these measures, the abnormal cervical cytology reporting rates significantly increased in large-scale test programs involving the CPS or LBC method, suggesting that cytopathologist training and QC programs significantly improve screening efficacy [9]. In this study, we further compared the 
screening efficiency of LBC testing alone, HR-HPV detection alone, and their combination in patients who subsequently received histological diagnoses of CIN1, CIN2/3, and ICC. All screening tests were performed over a 4-year period after the implementation of a systematic training and QC program. The aim of this study is to provide further scientific basis for the establishment of a systematic training and QC program, and to guide the selection of the optimal primary screening method for cervical cancer prevention in an underserved population from Shandong Province, China.

\section{Methods}

\section{Patient cohort}

JKD provides clinicopathological services to more than 900 hospitals, physical examination centers, and community clinics throughout Shandong Province. After a formal approval by KD's ethics review board, patients with histological diagnoses of CIN1, CIN2/3, and ICC were identified from the pathology databases of JKD over a 4-year period from January 2014 to December 2017. In this study, we included only the cytology and/or HR-HPV testing results obtained within the 3 months preceding a CIN1 diagnosis or within the 6 months preceding a CIN2/3 or ICC diagnosis. The majority of the cases included in this study were collected from local hospitals, physical examination centers, and community clinics that serve mainly suburban and rural areas, where a large number of clinicians are not specially trained or qualified. Cytology alone, HR-HPV testing alone, and co-testing with cytology and HR-HPV testing were all used as screening modalities. The diagnoses of CIN1, CIN2/3, and ICC were rendered by histopathological examinations, including cervical biopsy, endocervical curettage, diagnostic excisional procedures, and hysterectomy. All LBC and histopathological examinations were performed at the Pathology Department of JKD, while HR-HPV detection was performed at the Molecular Department of JKD. Cytology and/or HR-HPV testing data from other hospitals or laboratories were not included in this study.

\section{Cytology preparation and interpretation}

LBC preparation was carried out strictly in accordance with the manufacturers' instructions $[9,27,28]$. All cytological examinations were reported using the terminology of the 2001 Bethesda System. The cytological interpretations were divided as follows: unsatisfactory specimen, negative for intraepithelial lesion or malignancy (NILM), atypical cells of undetermined significance (ASC-US), low-grade squamous intraepithelial lesion (LSIL), atypical squamous cells-cannot exclude high-grade squamous intraepithelial lesion (ASC-H), high-grade squamous intraepithelial lesion (HSIL), atypical glandular cells (AGC), and cervical cancer cells. We conducted a rigorous and systematic training and QC program for cytology processes, as we have previously reported $[9,10]$.

\section{HR-HPV testing}

JKD has established the largest standardized molecular laboratory in Shandong Province and has obtained the International Organization for Standardization certification for molecular diagnosis to ensure the diagnostic accuracy of molecular testing. Nearly 200,000 samples are tested for HPV every year in the JKD laboratory. In this study, all HR-HPV tests were carried out in the standardized molecular laboratory of JKD by using one of two methods: Hybrid Capture 2 (HC2; Qiagen, Hinden, Germany) and HPV genotyping (Yanengbio, Shenzhen, China) [27-29]. The HC2 assay is an in vitro nucleic acid hybridization method, which can semiquantitatively test for 13 HR-HPV genotypes (i.e., 16, 18, $31,33,35,39,45,51,52,56,58,59$, and 68). The HPV genotyping assay is an in vitro diagnostic kit using PCRreverse dot blot hybridization measurement, and it can detect 14 HR-HPV genotypes (i.e., 16, 18, 31, 33, 35, 39, $45,51,52,56,58,59,66$, and 68) and 9 low- or uncertain-risk HPV genotypes $(6,11,42,43,53,73,81$, 82 , and 83). Only infections with one or more of the 14 HR-HPV genotypes were considered as a positive HRHPV test result in this study.

\section{Statistical analysis}

Statistical analysis was conducted using SPSS software (version 19.0, IBM Co., Chicago, Illinois, USA). The Pearson $\chi^{2}$ test was used to compare differences in categorical data, and the one-way analysis of variance were used to compare differences in continuous data, with the Bonferroni test being carried out where appropriate. $P<0.05$ was considered statistically significant.

\section{Results}

\section{Patient characteristics}

During the 4-year study period, CIN1, CIN2/3, and ICC were histologically diagnosed in a total of 1829 patients, 2309 patients, and 680 patients, respectively, who had undergone prior cytology and/or HR-HPV testing (Table 1). Of the 680 patients with ICC, $585(86.0 \%)$ patients had squamous cell carcinoma, $86(12.6 \%)$ patients had adenocarcinoma, and $9(1.3 \%)$ patients had adenosquamous carcinoma. The average age of the ICC patients was significantly higher than those of the CIN1 $(P<0.001)$ and $C I N 2 / 3$ patients $(P<0.001)$, but no significant difference was observed between the average ages of the CIN 1 and $C I N 2 / 3$ patients $(P=0.846)$. In each of these three groups, no significant differences in 
average age were found between the different screening modalities.

\section{Results of prior cytology alone}

The detailed results of cytology alone are summarized in Table 2. The average interval between histological diagnosis and cytological testing was 21.8 days (range, 0-90 days), 20.5 days (range, $0-166$ days), and 12.6 days (range, 0-160 days) in the CIN1, CIN2/3, and ICC groups, respectively. Overall, the rate of abnormal cytological findings was significantly higher in the CIN2/3 group $(97.2 \%)$ than in the CIN1 $(92.9 \%, P<0.001)$ and ICC groups $(89.1 \%, P<0.001)$. However, this rate did not differ between the CIN1 and ICC groups $(P=0.083)$. LSIL was the most common abnormal cytological result in the CIN1 group (reported in $65.4 \%$ of patients), followed by ASC-US (20.5\%), HSIL (5.2\%), and ASC-H (1.8\%). In contrast, HSIL was the most common abnormal cytological result in the CIN2/3 (52.7\%) and ICC (48.3\%) groups. The other abnormal results in the CIN2/ 3 group were LSIL (24.1\%), ASC-H (12.2\%), ASC-US $(8.0 \%)$, cancer cells $(0.2 \%)$, and unsatisfactory cellularity $(0.1 \%)$. In the ICC group, cancer cells $(16.1 \%)$ was the second most common cytological interpretation, followed by ASC-H (16.1\%), NILM (8.3\%), AGC (7.0\%), ASC-US (5.2\%), unsatisfactory cellularity (2.6\%), and LSIL (0.4\%).

\section{Results of prior HR-HPV testing alone}

Among patients who underwent only HR-HPV testing using the $\mathrm{HC} 2$ assay, the final histological diagnoses were as follows: CIN1, 309 (53.8\%) patients; CIN2/3, 499 (55.1\%) patients; and ICC, 69 (31.4\%) patients (Table 3). The remaining patients who underwent HR-HPV testing alone underwent genotyping tests. The average interval between histological diagnosis and HR-HPV testing was 9.8 days (range, $0-90$ days), 9.3 days (range, $0-146$ days), and 6.5 days (range, $0-157$ days) in the CIN1, CIN2/3, and ICC groups, respectively. The overall HR-HPV prevalence in the CIN2/3 (95.4\%) and ICC (92.7\%) groups was similar to each other $(P=0.112)$ but significantly higher than that in the CIN1 group $(79.9 \%$, all $P<0.001)$. The rate of positive HR-HPV results was
Table 2 Results of prior cytology alone

\begin{tabular}{|c|c|c|c|c|c|c|}
\hline \multirow{2}{*}{$\begin{array}{l}\text { Cytological } \\
\text { interpretation }\end{array}$} & \multicolumn{2}{|c|}{ CIN1 } & \multicolumn{2}{|c|}{ CIN2/3 } & \multicolumn{2}{|l|}{ ICC } \\
\hline & No. & $\%$ & No. & $\%$ & No. & $\%$ \\
\hline NILM & 40 & 7.1 & 24 & 2.7 & 19 & 8.3 \\
\hline Unsatisfactory & 0 & 0.0 & 1 & 0.1 & 6 & 2.6 \\
\hline ASC-US & 115 & 20.5 & 70 & 8.0 & 12 & 5.2 \\
\hline ASC-H & 10 & 1.8 & 107 & 12.2 & 28 & 12.2 \\
\hline LSIL & 366 & 65.4 & 212 & 24.1 & 1 & 0.4 \\
\hline HSIL & 29 & 5.2 & 463 & 52.7 & 111 & 48.3 \\
\hline AGC & 0 & 0.0 & 0 & 0.0 & 16 & 7.0 \\
\hline Cancer cells & 0 & 0.0 & 2 & 0.2 & 37 & 16.1 \\
\hline Total & 560 & 100.0 & 879 & 100.0 & 230 & 100.0 \\
\hline
\end{tabular}

CIN cervical intraepithelial neoplasia, ICC invasive cervical cancer, NILM negative for intraepithelial lesion or malignancy, ASC-US atypical squamous cells of undetermined significance, ASC-H atypical squamous cells-cannot exclude HSIL, LSIL low-grade squamous intraepithelial lesion, HSIL high-grade squamous intraepithelial lesion, $A G C$ atypical glandular cells

similar for the two HPV testing methods in the CIN2/3 (HC2: $95.4 \%$ vs. genotyping: $95.3 \% ; P=0.996$ ) and ICC groups (HC2: $94.2 \%$ vs. genotyping: $92.1 \% ; P=0.569$ ). In the CIN1 group, however, $\mathrm{HC} 2$ testing resulted in a significantly higher rate of positive results than did genotyping $(85.1 \%$ vs. $76.0 \% ; P=0.003)$.

\section{Prior co-testing results}

Among the patients who underwent both cytology and HR-HPV co-testing, the histological diagnoses were as follows: CIN1, 556 patients; CIN2/3, 524 patients; and ICC, 230 patients. HC2 testing was performed in 194 (34.9\%) of the 556 CIN1 patients, 213 (40.6\%) of the 524 CIN2/3 patients, and $41(17.8 \%)$ of the 230 ICC patients; the remaining patients underwent HPV genotyping (Table 4). The average interval between histological diagnosis and co-testing was 15.8 days (range, 0-90 days), 17.3 days (range, 0-127 days), and 10.4 days (range, $0-80$ days) in the CIN1, CIN2/3, and ICC groups, respectively. The rate of abnormal cytological results was significantly lower in the CIN1 group (87.9\%, $489 / 556)$ than in the CIN2/3 $(92.7 \%, 486 / 524 ; P=0.008)$ and ICC groups $(93.5 \%, 215 / 230 ; P=0.021)$. The overall HR-HPV prevalence in the CIN2/3 (92.7\%) and ICC

Table 1 Ages of patients who underwent cytology alone, HR-HPV testing alone, and co-testing prior to the diagnoses of CIN1, CIN2/3, and ICC

\begin{tabular}{|c|c|c|c|c|c|c|}
\hline \multirow[t]{2}{*}{ Screening modality } & \multicolumn{2}{|l|}{ CIN1 } & \multicolumn{2}{|l|}{$\mathrm{CIN} 2 / 3$} & \multicolumn{2}{|l|}{ ICC } \\
\hline & No. (\%) & Age, average (range), y & No. (\%) & Age, average (range), y & No. (\%) & Age, average (range), y \\
\hline Cytology alone & $560(30.6)$ & $41.0(18-64)$ & $879(38.1)$ & $42.5(16-71)$ & $230(33.8)$ & $49.0(27-85)$ \\
\hline HR-HPV testing alone & $713(39.0)$ & $41.5(14-66)$ & $906(39.2)$ & $41.1(20-67)$ & $220(32.4)$ & $47.9(25-88)$ \\
\hline Cytology and HR-HPV co-testing & $556(30.4)$ & $40.4(17-70)$ & $524(22.7)$ & $39.8(18-72)$ & $230(33.8)$ & $47.9(28-87)$ \\
\hline Total & $1829(100)$ & $41.0(17-70)$ & $2309(100)$ & $41.3(16-72)$ & $680(100)$ & $48.3(25-88)$ \\
\hline
\end{tabular}

CIN cervical intraepithelial neoplasia, ICC invasive cervical cancer, HR-HPV high-risk human papillomavirus 
Table 3 Results of prior HR-HPV testing alone

\begin{tabular}{|c|c|c|c|c|c|c|}
\hline \multirow{2}{*}{$\begin{array}{l}\text { HPV test } \\
\text { method }\end{array}$} & \multicolumn{2}{|l|}{ CIN1 $(\boldsymbol{n}=713)$} & \multicolumn{2}{|c|}{$\mathrm{CIN} 2 / 3(\boldsymbol{n}=906)$} & \multicolumn{2}{|l|}{ ICC $(\boldsymbol{n}=220)$} \\
\hline & Negative (\%) & Positive (\%) & Negative (\%) & Positive (\%) & Negative (\%) & Positive (\%) \\
\hline HC2 test & $46(14.9)$ & $263(85.1)$ & $23(4.6)$ & 476 (95.4) & $4(5.8)$ & $65(94.2)$ \\
\hline Genotyping & $97(24.0)$ & 307 (76.0) & $19(4.7)$ & 388 (95.3) & $12(7.9)$ & $139(92.1)$ \\
\hline Total & $143(20.1)$ & $570(79.9)$ & $42(4.6)$ & $864(95.4)$ & $16(7.3)$ & $204(92.7)$ \\
\hline
\end{tabular}

HR-HPV high-risk human papillomavirus, CIN cervical intraepithelial neoplasia, ICC invasive cervical cancer, HC2 Hybrid Capture 2

(94.8\%) groups was similar to each other $(P=0.718)$ but significantly higher than that in the CIN1 group $(72.8 \%$, all $P<0.001)$. HC2 testing and genotyping detection had similar rates of HR-HPV detection in the CIN1 $(76.3 \%$ vs. $71.0 \%$, respectively; $P=0.181), \mathrm{CIN} 2 / 3(93.9 \%$ vs. 92.0\%, $P=0.401)$, and ICC (97.6\% vs. $94.2 \%, P=0.377)$ groups. The rate of abnormal findings on co-testing was significantly higher in the CIN2/3 $(99.8 \%, P<0.001)$ and ICC groups $(99.6 \%, P=0.003)$ than in the CIN1 group
(95.3\%), but the rates were similar in the former two groups $(P=0.549)$.

\section{Comparison of different screening modalities}

A comparison of the sensitivities of cytology alone, HRHPV testing alone, and co-testing with both cytology and HR-HPV detection is shown in Table 5. In the CIN1 group, cytology alone (92.9, 95\% confidence interval [CI]: 90.7-95.0) was significantly more sensitive than

Table 4 Results of cytology and HR-HPV co-testing

\begin{tabular}{|c|c|c|c|c|c|c|}
\hline \multirow{3}{*}{$\begin{array}{l}\text { Cytological } \\
\text { interpretation }\end{array}$} & \multicolumn{4}{|c|}{ HR-HPV testing } & \multirow{2}{*}{\multicolumn{2}{|c|}{ Total }} \\
\hline & \multicolumn{2}{|l|}{$\mathrm{HC} 2$ testing } & \multicolumn{2}{|l|}{ Genotyping } & & \\
\hline & Negative (\%) & Positive (\%) & Negative (\%) & Positive (\%) & Negative (\%) & Positive (\%) \\
\hline \multicolumn{7}{|l|}{ CIN1 $(n=556)$} \\
\hline NILM & $3(1.5)$ & $14(7.2)$ & $23(6.4)$ & $27(7.5)$ & $26(4.7)$ & $41(7.4)$ \\
\hline ASC-US & $20(10.3)$ & 49 (25.3) & $40(11.0)$ & $91(25.1)$ & $60(10.8)$ & $140(25.2)$ \\
\hline ASC-H & $2(1.0)$ & $4(2.1)$ & $3(0.8)$ & $3(0.8)$ & $5(0.9)$ & $7(1.3)$ \\
\hline LSIL & $18(9.3)$ & 79 (40.7) & $38(10.5)$ & $130(35.9)$ & $56(10.1)$ & 209 (37.6) \\
\hline HSIL & $3(1.5)$ & $2(1.0)$ & $1(0.3)$ & $6(1.7)$ & $4(0.7)$ & $8(1.4)$ \\
\hline Total & 46 (23.7) & $148(76.3)$ & $105(29.0)$ & $257(71.0)$ & $151(27.2)$ & $405(72.8)$ \\
\hline \multicolumn{7}{|l|}{ CIN2/3 $(n=524)$} \\
\hline NILM & $0(0)$ & $12(5.6)$ & $1(0.3)$ & $25(8.0)$ & $1(0.2)$ & $37(7.1)$ \\
\hline ASC-US & $2(0.9)$ & $33(15.5)$ & $7(2.3)$ & $53(17.0)$ & $9(1.7)$ & 86 (16.4) \\
\hline ASC-H & $2(0.9)$ & $35(16.4)$ & $2(0.6)$ & $22(7.1)$ & $4(0.8)$ & $57(10.9)$ \\
\hline LSIL & $4(1.9)$ & $52(24.4)$ & $6(1.9)$ & 74 (23.8) & $10(1.9)$ & $126(24.0)$ \\
\hline $\mathrm{HSIL}$ & $5(2.3)$ & 68 (31.9) & $9(2.9)$ & $112(36.0)$ & $14(2.7)$ & $180(34.4)$ \\
\hline Total & $13(6.1)$ & 200 (93.9) & $25(8.0)$ & $286(92.0)$ & $38(7.3)$ & $486(92.7)$ \\
\hline \multicolumn{7}{|l|}{ ICC $(n=230)$} \\
\hline NILM & $0(0)$ & $3(7.3)$ & $1(0.5)$ & $6(3.2)$ & $1(0.4)$ & $9(3.9)$ \\
\hline Unsatisfactory & $0(0)$ & $0(0)$ & $0(0)$ & $5(2.6)$ & $0(0)$ & $5(2.2)$ \\
\hline ASC-US & $1(2.4)$ & $3(7.3)$ & $3(1.6)$ & $15(7.9)$ & $4(1.7)$ & $18(7.8)$ \\
\hline ASC-H & $0(0)$ & $10(24.4)$ & $1(0.5)$ & $29(15.3)$ & $1(0.4)$ & 39 (17.0) \\
\hline LSIL & $0(0)$ & $0(0)$ & $0(0)$ & $1(0.5)$ & $0(0)$ & $1(0.4)$ \\
\hline HSIL & $0(0)$ & $18(43.9)$ & $1(0.5)$ & $100(52.9)$ & $1(0.4)$ & $118(51.3)$ \\
\hline AGC & $0(0)$ & $0(0)$ & $2(1.1)$ & $3(1.6)$ & $2(0.9)$ & $3(1.3)$ \\
\hline Cancer cells & $0(0)$ & $6(14.6)$ & $3(1.6)$ & $19(10.1)$ & $3(1.3)$ & $25(10.9)$ \\
\hline Total & $1(2.4)$ & 40 (97.6) & $11(5.8)$ & $178(94.2)$ & $12(5.2)$ & $218(94.8)$ \\
\hline
\end{tabular}

HR-HPV high-risk human papillomavirus, CIN cervical intraepithelial neoplasia, ICC invasive cervical cancer, NILM negative for intraepithelial lesion or malignancy, ASC-US atypical squamous cells of undetermined significance, ASC-H atypical squamous cells-cannot exclude HSIL, LSIL low-grade squamous intraepithelial lesion, HSIL high-grade squamous intraepithelial lesion, AGC atypical glandular cells, HC2 Hybrid Capture 2 
Table 5 Comparison of cytology alone, HR-HPV testing alone, and co-testing

\begin{tabular}{|c|c|c|c|c|c|c|}
\hline \multirow[t]{2}{*}{ Screening modality } & \multicolumn{2}{|l|}{ CIN1 $(\boldsymbol{n}=1829)$} & \multicolumn{2}{|c|}{ CIN2/3 $(\boldsymbol{n}=2308)$} & \multicolumn{2}{|l|}{ ICC $(\boldsymbol{n}=680)$} \\
\hline & Sensitivity, \% & $95 \% \mathrm{Cl}$ & Sensitivity, \% & $95 \% \mathrm{Cl}$ & Sensitivity, \% & $95 \% \mathrm{Cl}$ \\
\hline Cytology alone & $92.9(520 / 560)$ & $90.7-95.0$ & $97.2(854 / 879)$ & $96.1-98.3$ & $89.1(205 / 230)$ & $85.1-93.2$ \\
\hline HR-HPV testing alone & $79.9(570 / 713)$ & $77.0-82.9$ & $95.4(864 / 906)$ & $94.0-96.7$ & $92.7(204 / 220)$ & $89.3-96.2$ \\
\hline Co-testing & $95.3(530 / 556)$ & $93.6-97.1$ & $99.8(523 / 524)$ & 99.4-100 & $99.6(229 / 230)$ & $98.7-100$ \\
\hline
\end{tabular}

HR-HPV high-risk human papillomavirus, CIN cervical intraepithelial neoplasia, ICC invasive cervical cancer, $\mathrm{Cl}$ confidence interval

HR-HPV testing alone (79.9, 95\% CI: 77.0-82.9; $P<$ 0.001). In the same group, co-testing (95.3, 95\% CI: 93.6-97.1) was significantly more sensitive than HRHPV testing alone $(P<0.001)$ but only slightly more sensitive than cytology alone $(P=0.105)$. In the $C I N 2 / 3$ group, the sensitivity of cytology alone (97.2, 95\% CI: 96.1-98.3) was marginally higher than that of HR-HPV testing alone (95.4, 95\% CI: 94.0.0-96.7; $P=0.062$ ). However, the sensitivity of the combination (99.8, 95\% CI: 99.4-100) was significantly higher than that of cytology alone $(P=0.001)$ and HR-HPV testing alone $(P<0.001)$. In the ICC group, HR-HPV testing alone (92.7, 95\% CI: 89.3-96.2) was slightly, but not significantly more sensitive than cytology alone (89.1, 95\% CI: 85.1-93.2, $P=0.185)$, while co-testing (99.6, 95\% CI: 98.7-100) was significantly more sensitive than cytology alone $(P<0.001)$ and HR-HPV testing alone $(P<0.001)$. Furthermore, when 6 cases of unsatisfactory cytological interpretation were excluded (these cases needed to be resampled according to the guidelines $[12,15])$, cytology alone (91.5\% [205/224], 95\% CI: 87.9-95.2) had very similar screening efficiency to HR-HPV testing alone $(P=0.636)$.

\section{Discussion}

The present study demonstrated that cervical cytology alone and HR-HPV testing alone had similar efficiency for CIN2/3 and ICC detection, while cervical cytology alone was significantly more sensitive than HR-HPV testing alone for CIN1 detection. Additionally, the study showed that compared to either method alone, combined co-testing could further slightly increase the screening efficiency for CIN1, CIN2/3, and ICC. All of the above data were obtained after the implementation of a systematic training and QC program for cervical cytology screening in Shandong Province, China, and the population of women tested had not previously undergone intensive screening. These results suggest that a systematic cervical cytology training and QC program can improve the screening efficiency of cervical cytology for the detection of CIN1, CIN2/3, and ICC, so that it is equivalent to or even slightly higher than that of HRHPV detection.

A biopsy diagnosis of a CIN2/3 or worse lesion is the clinical threshold leading to ablative or excisional therapy. The treatment of CIN1 lesions, which have substantial rates of spontaneous regression, is discouraged, particularly in adolescents $[15,30]$. However, it is imperative to closely follow CIN1 patients up, as the cumulative incidence of $\mathrm{CIN} 2 / 3$ or worse lesions is very high among CIN1 patients, especially among those with HRHPV infection [30, 31]. Cytology and/or HR-HPV testing is recommended for follow-up evaluations $[15,30]$. Thus far, limited data are available on the screening effectiveness of cytology and/or HR-HPV testing for the detection of CIN1. In our study, the sensitivity of cytology alone (92.9\%) was significantly higher than that of HR-HPV testing alone (79.9\%) in the CIN1 group. Furthermore, adding HR-HPV testing to cytology (95.3\%) provided only a small and statistically insignificant increase in the screening sensitivity for CIN1 detection. In a large-scale summary of meta-analyses, Arbyn et al. [32] reported that a reflex $\mathrm{HC} 2$ test does not have a significantly higher sensitivity and has a significantly lower specificity than a repeat Pap smear for the triaging of women with LSIL. About $20-30 \%$ of patients with CIN1 test positive for only low-risk $\operatorname{HPV}[32,33]$, and this may explain why HR-HPV testing alone had a low screening efficacy and why adding HR-HPV testing to cytology did not significantly improve the screening effectiveness for CIN1 detection. However, clinical studies have found that women with LSIL accompanied with low-risk HPV infection or no HPV infection rarely progress to $\mathrm{CIN} 2 / 3$ or worse lesions [31, 34]. Given that CIN1 progression is closely related to HR-HPV infection, HR-HPV testing, like cervical cytology, has high clinical utility in CIN1 screening.

Our data revealed that the rate of abnormal results was marginally higher for cytology alone (97.2\%) than for HR-HPV testing alone (95.4\%) in the CIN2/3 group, which is comparable to the data from the Cytopathology Department of Guangzhou KD (GKD), which is the headquarters of KD with full CAP certification in China [35]. GKD is in strict conformity with the laboratory workload standards and QC practices issued in the CAP Laboratory Accreditation Program checklists [10]. According to the GKD data, 93.1\% patients had abnormal cytological results, and $91.7 \%$ patients had positive HRHPV testing results within 6 months prior to the histological diagnosis of CIN2/3. Another study from West 
China also reported that the rate of positive results was significantly higher for cytology (95.7\%) than for HRHPV testing (89.9\%) in 1094 CIN2/3 patients [36]. The screening results of CIN2/3 patients in this study and the two other studies $[35,36]$ from China are inconsistent with the results from dozens of randomized, controlled trials, which found that HR-HPV testing is more sensitive than cytology for identifying cervical cancer and its precursors during population screening [14, 3739]. Additionally, a study of 14,261 cases from multiple US clinical centers [40] reported a $91.4 \%$ rate of positive results for cervical cytology and a $95.8 \%$ rate for HRHPV testing performed within 1 year prior to the histological diagnosis; these results are discordant with the above Chinese data. This difference might be attributed to the fact that the populations from China had very limited prior screening, whereas the populations of developed countries largely undergo routine periodic screening. For those women who were never or rarely screened, CIN2/3 lesions might be diagnosed at much larger sizes than the lesions detected in women who underwent regular screening [41]. Therefore, more exfoliated neoplastic cells can be collected for making a definite interpretation, and the screening effectiveness of cytology might be higher in the underserved women than in the routinely screened women.

The present screening data showed that HR-HPV testing alone $(92.7 \%)$ was only slightly more sensitive than cytology alone $(89.1 \%)$ and without statistical significance in ICC patients, especially when cases of unsatisfactory interpretation had been excluded (91.5\%). One retrospective study of GKD data [42] examined the screening results of 155 Chinese women who were diagnosed with ICC within 1 year after undergoing cervical cancer screening. The results of the GKD study showed that cervical cytology was significantly more sensitive than HR-HPV testing, with the rate of negative results being $1.9 \%$ for cytology and $9.7 \%$ for HR-HPV testing [42]. In contrast, the rate of negative cytological findings was as high as $15.5 \%$ among patients subsequently diagnosed with ICC in both the first (238 patients) [43] and second (161 patients) [36] largest women's hospitals in China, in which no systematic training and QC processes were implemented. The rates of negative HRHPV testing resulting in these two hospitals were 15.5 and $12.4 \%$, respectively, which are much higher than the $7.3 \%$ rate in the present study and the $9.7 \%$ rate reported in the GKD study [42]. This difference in the screening efficacy of cytology and HR-HPV testing for ICC detection might be attributed to the high-quality cytology services offered after the establishment of systemic training and QC programs at JKD and GKD. Interestingly, a high rate of negative cytological results of $13.7 \%$, and a concurrent $10.8 \%$ rate of negative HR-HPV testing were reported in a large US study involving the co-testing of 600 patients who were eventually diagnosed with ICC [44]. As mentioned above, one possible explanation might be due to differences in populations that did or did not undergo regular screening. For the largely underserved Chinese women, ICC lesions might be diagnosed at much larger sizes and/or at later stages [41].

Co-testing has been shown to not only detect significantly more $\mathrm{CIN} 2 / 3$ or worse lesions but also results in significantly lower rates of ICC and its precursor lesions in subsequent rounds of screening [13, 14, 17]. In the present study, we also found that the cytology and HRHPV combination (99.8\%) had significantly higher sensitivity than either cytology alone or HR-HPV testing alone in CIN2/3 detection, which is consistent with the three aforementioned retrospective studies from China $[35,36]$ and the US [40], which reported rates of positive co-testing results in 98.1, 99.6, and $99.4 \%$ of cases, respectively. Furthermore, co-testing (99.6\%) in this study was significantly more sensitive than cytology alone and HR-HPV testing alone for ICC detection. These results are in accordance with retrospective studies from other daily clinical practices [36, 40, 42-44] and the aforementioned randomized controlled trials [13, 14, 17].

As a cervical cancer screening method, cytology has several advantages, such as simple preparation, low infrastructure requirements, and cost-effectiveness. In addition, the detection of different cytological abnormalities is helpful for the clinical triage of patients. Even now, cervical cytology (both LBC and CPS) is widely used for the large-scale screening and follow-up of highrisk groups, especially, in areas with poor economic conditions in China $[9,10,25]$. Our present data and those of several other Chinese studies [35, 36, 42, 43, 45] have demonstrated that cytology has similar or even slightly higher efficiency than HR-HPV testing for CIN $2 / 3$ and ICC screening. However, the interpretation of cervical cytological results involves a certain degree of subjectivity and requires specially trained and qualified cytotechnologists and/or cytopathologists. Moreover, strict QC standards are required to ensure the accuracy of interpretation. Developed countries have established wellorganized cervical cytology training and QC programs to guarantee the efficiency of cytological screening $[4,5]$. The data of this study and the GKD data [35, 42] indicate that systematic training and QC programs can markedly increase the screening effectiveness of cervical cytology in China. Of note, many challenges still exist in using cytology as a first-line method for cervical cancer screening in China. Even in Shandong Province, which has a relatively developed economy, systematic training programs for cytopathologists and standardized QC systems have not yet been widely established, and cervical cancer screening is not routinely carried out. A recent 
population-based, prospective study [46] from China reported a screening sensitivity of less than $71.1 \%$ for cervical cytology in the detection of CIN $2 / 3$ and ICC. These factors are the main reason for the high incidence and mortality of cervical cancer in China [23, 24].

In addition to accelerating the training of cytopathologists and establishing a cytological QC system to improve the efficiency of cervical cytology, it is necessary to seek alternative screening methods and gradually reduce the dependence on qualified cytopathologists. Compared with cytological screening, HR-HPV detection is easy to automate and establish QC processes for, which would ensure the accuracy of screening. Therefore, HR-HPV testing as a firstline screening method for cervical cancer has high application value in areas where there is a lack of qualified cytopathologists in China. However, we should also note that HR-HPV detection alone as a first-line screening method for cervical cancer has some limitations. Usually, most of the HPV load will automatically be eliminated by the host immune system within $8-10$ months $[2,3]$. Only a few persistent infections have the possibility of developing into cervical precancerous lesions and cervical cancer $[2,3]$. This results in an inherent drawback in using HRHPV detection as a first-line screening method [47]. Clinical studies $[13,46]$ have confirmed that the specificity and positive predictive value of HR-HPV testing are lower than those of cytological screening. According to the requirements of the American Society for Colposcopy and Cervical Pathology, women with positive HR-HPV test results need further colposcopy or cytology [15]. A recent retrospective analysis of 94,489 HPV genotyping results [29] showed that the rate of positive HR-HPV testing results was $24.2 \%$ in women from Shandong Province. Such a large number of HR-HPV-positive women, in most of whom the viral infection may be naturally eliminated by their own immune system, complicates the formulation of further treatment plans and raises concerns about excessive colposcopy and treatments for selflimiting HPV infections. Therefore, it is necessary to find new markers to separate out the cases of CIN2/3 or cervical cancer in HR-HPV-positive women and improve the efficiency of HR-HPV screening. In addition, HPV testing costs were about three times as much as cytology, and would place a heavy economic burden on underdeveloped regions, especially in suburban and rural areas. Moreover, a dozen HPV detection kits are currently used in clinical practice in China. The clinical validity and utility of these kits have not been fully verified using large-scale clinical trials, leading to a wide spectrum of difference in the first-line screening performance of these kits for cervical cancer.

\section{Conclusions}

In conclusion, the results of this study show that the screening effectiveness of cervical cytology after the implementation of a systematic training and QC program was similar or even slightly higher than that of HR-HPV testing for the detection of CIN1, CIN2/3, and ICC in a largely unscreened population from Shandong Province, China. The experience of JKD can provide a good example to create training programs for cytopathologists and QC standards for cervical cancer screening in this region. In addition, new screening methods, such as HRHPV detection, can be adopted as a favored alternative to cervical cytology, which may gradually reduce the dependence on cytological screening and rapidly popularize cervical cancer screening among women in low-resource settings such as rural areas from Shandong Province, China.

\section{Abbreviations \\ JKD: Jinan KingMed Diagnostics; QC: Quality control; CIN: Cervical intraepithelial neoplasia; ICC: Invasive cervical cancer; CPS: Conventional Papanicolaou smear; LBC: Liquid-based cytology; HR-HPV: high-risk human papillomavirus; KD: KingMed Diagnostics; CAP: College of American Pathologists; HC2 assay: Hybrid Capture 2 assay; NILM: Negative for intraepithelial lesion or malignancy; ASC-US: Atypical squamous cells of undetermined significance; LSIL: Low-grade squamous intraepithelial lesion; HSIL: High-grade squamous intraepithelial lesion; ASC-H: Atypical squamous cells—cannot exclude HSIL; AGC: Atypical glandular cells; PCR: Polymerase chain reaction; GKD: Guangzhou KingMed Diagnostics}

\section{Acknowledgements}

Not applicable.

\section{Authors' contributions}

All of the authors had full access to all of the data (including the statistical reports and tables) in the study and taking responsibility for the content of the manuscript. DBQ conceived and designed the experiments. DMZ, LRZ, FXX, DZP, JW, LBJ and SDZ performed the case and sample collection, analysis, and interpretation of the data. DMZ and LRZ wrote the first draft of the paper. DBQ reviewed and approved the final manuscript. All authors have read and approved the final manuscript.

\section{Funding}

Not applicable.

\section{Availability of data and materials}

All relevant data are within the paper. The data underlying this study are available and researchers may submit data requests to the the corresponding author on reasonable request.

\section{Ethics approval and consent to participate}

This study was approved by the ethics review board of KingMed Diagnostics. Because the present study was an analysis of anonymous data, the ethics review board waived the need for patient consent. The patient data in this study were processed and published in strict accordance with the tenets of the Declaration of Helsinki, including the confidentiality and anonymity requirements.

Consent for publication

Not applicable.

Competing interests

None declared. 


\section{Author details}

'Department of Pathology, Jinan KingMed Diagnostics, Jinan 250101, Shandong Province, China. ${ }^{2}$ Department of Laboratory Medicine, Jinan KingMed Diagnostics, Jinan 250101, Shandong Province, China.

\section{Received: 7 February 2020 Accepted: 19 August 2020}

Published online: 26 August 2020

\section{References}

1. Ferlay J, Shin HR, Bray F, Forman D, Mathers C, Parkin DM. Estimates of worldwide burden of cancer in 2008: GLOBOCAN 2008. Int J Cancer. 2010; 127(12):2893-917

2. Crosbie EJ, Einstein MH, Franceschi S, Kitchener HC. Human papillomavirus and cervical cancer. Lancet. 2013;382(9895):889-99.

3. Wheeler CM. The natural history of cervical human papillomavirus infections and cervical cancer: gaps in knowledge and future horizons. Obstet Gynecol Clin N Am. 2013;40(2):165-76.

4. Gifford C, Coleman DV. Quality assurance in cervical cancer screening: results of a proficiency testing scheme for cytology laboratories in the north West Thames region. Cytopathology. 1994;5(4):197-206.

5. Wiener HG, Klinkhamer P, Schenck U, Arbyn M, Bulten J, Bergeron C, et al. European guidelines for quality assurance in cervical cancer screening: recommendations for cytology laboratories. Cytopathology. 2007;18(2):67-78.

6. Bergstrom R, Sparen $\mathrm{P}, \mathrm{Adami} \mathrm{HO}$. Trends in cancer of the cervix uteri in Sweden following cytological screening. Br J Cancer. 1999;81(1):159-66.

7. Arbyn M, Rebolj M, De Kok IM, Fender M, Becker N, O'Reilly M, et al. The challenges of organising cervical screening programmes in the 15 old member states of the European Union. Eur J Cancer. 2009;45(15):2671-8.

8. Adegoke $\mathrm{O}$, Kulasingam S, Virnig B. Cervical cancer trends in the United States: a 35-year population-based analysis. J Women's Health (Larchmt). 2012;21(10):1031-7.

9. Xie F, Li Z, Zhang L, Zhang H, Qi D, Zhao D, et al. Systemic cervical cytology training and quality control programs can improve the interpretation of Papanicolaou tests. J Am Soc Cytopathol. 2019;8(1):27-33.

10. Zheng B, Austin RM, Liang X, Li Z, Chen C, Yan S, et al. Bethesda system reporting rates for conventional Papanicolaou tests and liquid-based cytology in a large Chinese, College of American Pathologists-certified independent medical laboratory: analysis of 1394389 Papanicolaou test reports. Arch Pathol Lab Med. 2015;139(3):373-7.

11. de Sanjose S, Quint WG, Alemany L, Geraets DT, Klaustermeier JE, Lloveras B, et al. Human papillomavirus genotype attribution in invasive cervical cancer: a retrospective cross-sectional worldwide study. Lancet Oncol. 2010;11(11): 1048-56.

12. Wright TJ, Massad LS, Dunton CJ, Spitzer M, Wilkinson EJ, Solomon D. 2006 consensus guidelines for the management of women with abnormal cervical cancer screening tests. Am J Obstet Gynecol. 2007;197(4):346-55.

13. Katki HA, Kinney WK, Fetterman B, Lorey T, Poitras NE, Cheung L, et al. Cervical cancer risk for women undergoing concurrent testing for human papillomavirus and cervical cytology: a population-based study in routine clinical practice. Lancet Oncol. 2011;12(7):663-72.

14. Naucler P, Ryd W, Tornberg S, Strand A, Wadell G, Elfgren K, et al. Human papillomavirus and Papanicolaou tests to screen for cervical cancer. N Engl J Med. 2007:357(16):1589-97.

15. Massad LS, Einstein MH, Huh WK, Katki HA, Kinney WK, Schiffman M, et al. 2012 updated consensus guidelines for the management of abnormal cervical cancer screening tests and cancer precursors. Obstet Gynecol. 2013; 121(4):829-46.

16. Ronco G, Dillner J, Elfstrom KM, Tunesi S, Snijders PJ, Arbyn M, et al. Efficacy of HPV-based screening for prevention of invasive cervical cancer: follow-up of four European randomised controlled trials. Lancet. 2014;383(9916):524-32.

17. Rijkaart DC, Berkhof J, Rozendaal L, van Kemenade FJ, Bulkmans NW, Heideman DA, et al. Human papillomavirus testing for the detection of high-grade cervical intraepithelial neoplasia and cancer: final results of the POBASCAM randomised controlled trial. Lancet Oncol. 2012;13(1):78-88.

18. Zhao FH, Lin MJ, Chen F, Hu SY, Zhang R, Belinson JL, et al. Performance of high-risk human papillomavirus DNA testing as a primary screen for cervical cancer: a pooled analysis of individual patient data from 17 populationbased studies from China. Lancet Oncol. 2010;11(12):1160-71.
19. Ronco G, Giorgi-Rossi P, Carozzi F, Confortini M, Dalla PP, Del MA, et al. Efficacy of human papillomavirus testing for the detection of invasive cervical cancers and cervical intraepithelial neoplasia: a randomised controlled trial. Lancet Oncol. 2010;11(3):249-57.

20. Castle PE, Stoler MH, Wright TJ, Sharma A, Wright TL, Behrens CM. Performance of carcinogenic human papillomavirus (HPV) testing and HPV16 or HPV18 genotyping for cervical cancer screening of women aged 25 years and older: a subanalysis of the ATHENA study. Lancet Oncol. 2011; 12(9):880-90.

21. Huh WK, Ault KA, Chelmow D, Davey DD, Goulart RA, Garcia FA, et al. Use of primary high-risk human papillomavirus testing for cervical cancer screening: interim clinical guidance. Gynecol Oncol. 2015;136(2):178-82.

22. Castle PE, de Sanjose S, Qiao YL, Belinson JL, Lazcano-Ponce E, Kinney W. Introduction of human papillomavirus DNA screening in the world: 15 years of experience. Vaccine. 2012;30(Suppl 5):F117-22.

23. Di J, Rutherford S, Chu C. Review of the cervical cancer burden and population-based cervical cancer screening in China. Asian Pac J Cancer Prev. 2015;16(17):7401-7.

24. Chen W, Zheng R, Baade PD, Zhang S, Zeng H, Bray F, et al. Cancer statistics in China, 2015. CA Cancer J Clin. 2016;66(2):115-32.

25. Tao X, Austin RM, Kong L, Sun Q, Lv Q, Xu H, et al. Nationwide survey of cervical cytology laboratory practices in China. J Am Soc Cytopathol. 2019; 8(5):250-7.

26. Wang SM, Qiao YL. Implementation of cervical cancer screening and prevention in China--challenges and reality. Jpn J Clin Oncol. 2015;45(1):7-11.

27. Xie F, Zhang L, Zhao D, Wu X, Wei M, Zhang X, et al. Prior cervical cytology and high-risk HPV testing results for 311 patients with invasive cervical adenocarcinoma: a multicenter retrospective study from China's largest independent operator of pathology laboratories. BMC Infect Dis. 2019;19(1):962.

28. Zhang $L$, Xie F, Wang $X$, Peng D, Bi C, Jiang $L$, et al. Previous cervical cytology and high-risk human papillomavirus testing in a cohort of patients with invasive cervical carcinoma in Shandong Province, China. Plos one. 2017;12(6):e180618.

29. Jiang L, Tian X, Peng D, Zhang L, Xie F, Bi C, et al. HPV prevalence and genotype distribution among women in Shandong Province, China: analysis of 94,489 HPV genotyping results from Shandong's largest independent pathology laboratory. PLoS One. 2019;14(1):e210311.

30. Wright TJ, Massad LS, Dunton CJ, Spitzer M, Wilkinson EJ, Solomon D. 2006 consensus quidelines for the management of women with cervical intraepithelial neoplasia or adenocarcinoma in situ. J Low Genit Tract Dis. 2007;11(4):223-39.

31. Hu S, Zhao F, Ma J, Wang X, Han J, Li A, et al. A prospective study on the prognosis of biopsy-confirmed cervical intraepithelial neoplasia grade 1 and the relationship with high-risk human papillomavirus. Chin J Prev Med. 2014:48(5):361-5.

32. Arbyn M, Sasieni P, Meijer CJ, Clavel C, Koliopoulos G, Dillner J. Chapter 9: clinical applications of HPV testing: a summary of meta-analyses. Vaccine. 2006:24(Suppl 3):S3-78.

33. Clifford GM, Rana RK, Franceschi S, Smith JS, Gough G, Pimenta JM. Human papillomavirus genotype distribution in low-grade cervical lesions: comparison by geographic region and with cervical cancer. Cancer Epidemiol Biomark Prev. 2005:14(5):1157-64.

34. Nobbenhuis MA, Helmerhorst TJ, van den Brule AJ, Rozendaal L, Voorhorst FJ, Bezemer PD, et al. Cytological regression and clearance of high-risk human papillomavirus in women with an abnormal cervical smear. Lancet. 2001;358(9295):1782-3.

35. Wu T, Chen X, Zheng B, Li J, Xie F, Ding X, et al. Previous Papanicolaou and hybrid capture 2 human papillomavirus testing results of 5699 women with histologically diagnosed cervical intraepithelial neoplasia 2/3. J Am Soc Cytopathol. 2019;8(4):206-11.

36. Jiang W, Marshall AR, Li L, Yang K, Zhao C. Extended human papillomavirus genotype distribution and cervical cytology results in a large cohort of chinese women with invasive cervical cancers and high-grade squamous intraepithelial lesions. Am J Clin Pathol. 2018;150(1):43-50.

37. Pan QJ, Hu SY, Zhang X, Ci PW, Zhang WH, Guo HQ, et al. Pooled analysis of the performance of liquid-based cytology in population-based cervical cancer screening studies in China. Cancer Cytopathol. 2013;121(9):473-82.

38. Mayrand MH, Duarte-Franco E, Rodrigues I, Walter SD, Hanley J, Ferenczy A, et al. Human papillomavirus DNA versus Papanicolaou screening tests for cervical cancer. N Engl J Med. 2007;357(16):1579-88. 
39. Kitchener HC, Almonte M, Thomson C, Wheeler P, Sargent A, Stoykova B, et al. HPV testing in combination with liquid-based cytology in primary cervical screening (ARTISTIC): a randomised controlled trial. Lancet Oncol. 2009:10(7):672-82

40. Blatt AJ, Kennedy R, Luff RD, Austin RM, Rabin DS. Comparison of cervical cancer screening results among 256,648 women in multiple clinical practices. Cancer Cytopathol. 2015;123(5):282-8.

41. Benard VB, Royalty J, Saraiya M, Rockwell T, Helsel W. The effectiveness of targeting never or rarely screened women in a national cervical cancer screening program for underserved women. Cancer Causes Control. 2015; 26(5):713-9.

42. Zheng B, Li Z, Griffith CC, Yan S, Chen C, Ding X, et al. Prior high-risk HPV testing and pap test results for 427 invasive cervical cancers in China's largest CAP-certified laboratory. Cancer Cytopathol. 2015;123(7):428-34.

43. Tao X, Griffith CC, Zhou X, Wang Z, Yan Y, Li Z, et al. History of high-risk HPV and pap test results in a large cohort of patients with invasive cervical carcinoma: experience from the largest women's hospital in China. Cancer Cytopathol. 2015;123(7):421-7.

44. Schiffman M, Kinney WK, Cheung LC, Gage JC, Fetterman B, Poitras NE, et al. Relative performance of HPV and cytology components of cotesting in cervical screening. J Natl Cancer Inst. 2018;110(5):501-8.

45. Pan QJ, Hu SY, Guo HQ, Zhang WH, Zhang X, Chen W, et al. Liquid-based cytology and human papillomavirus testing: a pooled analysis using the data from 13 population-based cervical cancer screening studies from China. Gynecol Oncol. 2014;133(2):172-9.

46. Xu H, Lin A, Shao X, Shi W, Zhang Y, Yan W. Diagnostic accuracy of high-risk HPV genotyping in women with high-grade cervical lesions: evidence for improving the cervical cancer screening strategy in China. Oncotarget. 2016; 7(50):83775-83.

47. Malila N, Leinonen M, Kotaniemi-Talonen L, Laurila P, Tarkkanen J, Hakama M. The HPV test has similar sensitivity but more overdiagnosis than the pap test--a randomised health services study on cervical cancer screening in Finland. Int J Cancer. 2013:132(9):2141-7.

\section{Publisher's Note}

Springer Nature remains neutral with regard to jurisdictional claims in published maps and institutional affiliations.

Ready to submit your research? Choose BMC and benefit from:

- fast, convenient online submission

- thorough peer review by experienced researchers in your field

- rapid publication on acceptance

- support for research data, including large and complex data types

- gold Open Access which fosters wider collaboration and increased citations

- maximum visibility for your research: over $100 \mathrm{M}$ website views per year

At $\mathrm{BMC}$, research is always in progress.

Learn more biomedcentral.com/submissions 